\title{
A Framework for Integrating Mobility and Routing in Mobile Communication Networks
}

\author{
Michael M. Zavlanos, Alejandro Ribeiro, and George J. Pappas
}

\begin{abstract}
Most coordinated tasks performed by teams of mobile robots, require reliable communications between the members of the team. Therefore, task accomplishment requires that robots navigate their environment with their collective movement restricted to formations that guarantee integrity of the communication network. Maintaining this communication capability induces physical constraints on trajectories but also requires determination of communication variables like routes and transmitted powers. In this paper, we propose a novel framework to address this problem, in which continuous motion controllers based on potential fields interact with discrete optimization of the communication variables to result in a mutirobot network that ensures integrity of communications. Our definition of network integrity is defined as the ability of a network to support desired communication rates.
\end{abstract}

\section{INTRODUCTION}

Mobile robot networks have recently emerged as an inexpensive and reliable way to address a wide variety of tasks ranging from exploration, surveillance and reconnaissance, to cooperative construction and manipulation. Successful completion of these tasks requires efficient information exchange and coordination between members of the team.

Multi-hop communication in multi-robot systems has typically relied on constructs from graph theory, with proximity graphs gaining the most popularity. This is consistent with early approaches to wireless networking that used disk models to abstract the physical layer [1]. In this context, communication becomes equivalent to topological connectivity, defined as the property of a graph to transmit information between all pairs of its nodes. Preservation and control of topological connectivity has been widely studied recently with solutions ranging from maintaining all communication links [2] to least restrictive that allow links to be lost [3].

Although graphs provide a simple abstraction of interrobot communications, it has long being recognized that since links in a wireless network do not entail tangible connections, associating links with arcs on a graph can be somewhat arbitrary. Indeed, topological definitions of connectivity start by setting target signal strengths to draw the corresponding graph. Even small signal variations might result in dramatic differences in network topology [4].

The work of Michael M. Zavlanos is supported by the NSF CNS Grant \#1054604. The work of George J. Pappas and Alejandro Ribeiro is supported by the ONR HUNT MURI project.

Michael M. Zavlanos is with the Dept. of Mechanical Engineering, Stevens Institute of Technology, Hoboken, NJ 07030, USA michael.zavlanosestevens.edu. Alejandro Ribeiro and George J. Pappas are with the Dept. of Electrical and Systems Engineering, University of Pennsylvania, Philadelphia, PA 19104, USA \{aribeiro, pappasg\}@seas.upenn.edu.
In this paper, we employ a simple, yet effective, modification that relies on weighted graph models with weights that capture the packet error probability of each link [5]. When using reliabilities as link metrics it is possible to model routing and scheduling problems as optimization problems that accept link reliabilities as inputs [6]. The key idea proposed in this paper is to define connectivity in terms of communication rates and to use optimization formulations to describe optimal operating points of wireless networks [6]. Composition of discrete optimization of the communication variables with continuous motion control via appropriate barrier potentials that maintain desired communication rates, results in a distributed multi-robot hybrid system for which we show that desired communication rates are always guaranteed. We propose both a centralized and a distributed solution to the problem, the latter based on separability of the dual function and subgradient optimization. Since subgradient optimization ensures feasibility of the primal variables only asymptotically for static systems, it precludes verbatim use of those variables in barrier potentials in the physical domain. In fact, integration with node mobility gives rise to an infeasibility gap, which results in approximate communication guarantees that we explicitly analyze.

\section{OPTIMAL WIRELESS COMMUNICATIONS}

Consider a mobile network composed of $J$ robots and a fixed infrastructure with $K$ access points (APs). The robots move throughout an area of interest to accomplish an assigned task for which it is necessary to maintain reliable communications with the infrastructure. Due to, e.g., power constraints or an adverse propagation environment, robots collaborate to maintain a multihop network with the APs. Denote as $\mathbf{x}_{j}$ for $j=1, \ldots, J$ the position of the robots and $\mathbf{x}_{j}$ for $j=J+1, \ldots, J+K$ the position of the APs. The set of all positions $\mathbf{x}:=\left\{\mathbf{x}_{i}\right\}_{i=1}^{J+K}$ is referred to as the spatial configuration of the network. We model communication by a link reliability metric $R(\mathbf{x}, \mathbf{y})$ denoting the probability that a packet transmitted from a terminal located at position $\mathbf{x}$ is correctly decoded by a terminal at position $\mathbf{y}$. This function determines the probability $R_{i j} \triangleq R\left(\mathbf{x}_{i}, \mathbf{x}_{j}\right)$ with which a packet transmitted by node $i$ is correctly decoded by node $j$. Node $j$ is a robot if $j \leq J$ or an AP otherwise.

We further denote as $r_{i}$ the average rate at which robot $i$ delivers information to the APs. If robot $i$ can reach some of the APs, packets are directly conveyed to the corresponding AP. Otherwise, packets are routed to another robot for subsequent transmission. We model this process through the introduction of routing probabilities $T_{i j}$ denoting 


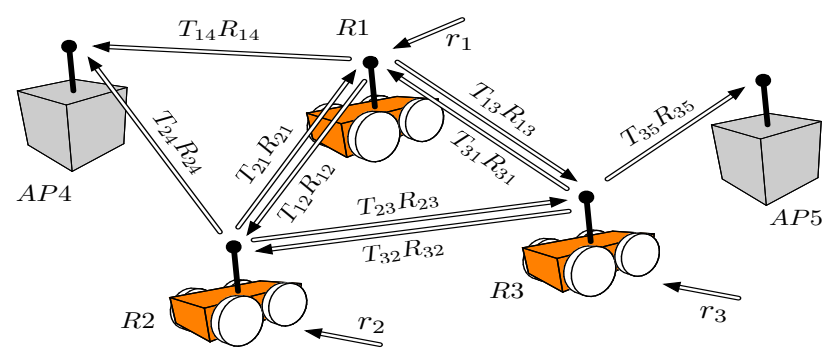

Fig. 1. Robotic network consisting of two access points (AP) and three robots (R). Shown are the packet rates $r_{i}$ generated by every robot as well as the rates $T_{i j} R\left(\mathbf{x}_{i}, \mathbf{x}_{j}\right)$ sent from robot $i$ and successfully decoded by robot $j$, where $T_{i j}$ is the probability that robot $i$ routes packets to robot $j$ and $R\left(\mathbf{x}_{i}, \mathbf{x}_{j}\right)$ is the reliability of the channel between robots $i$ and $j$.

the probability with which robot $i$ selects node $j$, a robot or an AP, as a destination of its transmitted packets; see Fig. 1.

Between the time of their generation or arrival from another robot and their transmission packets are stored in a queue. To ensure stability of these queues it suffices to require the average rate at which packets arrive at the $i$ th queue to be smaller than the average rate at which packets leave this queue. Thus, our interest is to determine routing probabilities $T_{i j}$ and rates $r_{i}$ that satisfy the inequality

$$
r_{i}+\sum_{j=1}^{J} T_{j i} R\left(\mathbf{x}_{j}, \mathbf{x}_{i}\right) \leq \sum_{j=1}^{J+K} T_{i j} R\left(\mathbf{x}_{i}, \mathbf{x}_{j}\right) .
$$

Any set of variables $\left\{r_{i}\right\}_{\forall i}$ and $\left\{T_{i j}\right\}_{\forall i, j}$ that satisfy the inequalities in (1) ensures information delivery. A basic requirement is that all robots can communicate with the infrastructure APs at least at a basal rate of $r_{i 0}$ packets per time unit. When this happens we say that we have network integrity as we formally define next.

Definition 1 (Network integrity) For configuration $\mathbf{x}$ and basal rates $r_{i 0}$, network integrity implies not only existence but also availability of rates $\left\{r_{i}\right\}_{\forall i}$ and routing variables $\left\{T_{i j}\right\}_{\forall i, j}$ for which the inequalities in (1) hold and $r_{i} \geq r_{i 0}$.

For a given spatial configuration $\left\{\mathbf{x}_{i}\right\}_{i=1}^{J+K}$ there might be various sets of variables that ensure network integrity. To select an element of this set we introduce strictly concave optimality criteria $U_{i}\left(r_{i}\right)$ and $V_{i j}\left(T_{i j}\right)$ measuring the value associated with variables $r_{i}$ and $T_{i j}$ respectively. The operating point is then selected as the solution of the problem

$$
\begin{aligned}
P_{\mathbf{x}}= & \max _{T_{i j}} \sum_{i=1}^{J} U_{i}\left(r_{i}\right)+\sum_{i=1}^{J} \sum_{j=1}^{J+K} V_{i j}\left(T_{i j}\right) \\
\text { s.t } \quad & r_{i}+\sum_{j=1}^{J} T_{j i} R\left(\mathbf{x}_{j}, \mathbf{x}_{i}\right) \leq \sum_{j=1}^{J+K} T_{i j} R\left(\mathbf{x}_{i}, \mathbf{x}_{j}\right), \\
& r_{i} \geq r_{i 0}, \quad \sum_{j=1}^{J} T_{i j} \leq 1,
\end{aligned}
$$

where the constraints are required for all $i \in\{1, \ldots, K\}$. To ensure network integrity for configuration $\mathbf{x}$, we need to find optimal routing probabilities $T_{i j}$ that solve the optimization problem in (2). This yields basal rates $r_{i 0}$ for all terminals, while assigning the remaining resources in a manner that is optimal in terms of utilities $U_{i}\left(r_{i}\right)$ and $V_{i j}\left(T_{i j}\right)$.
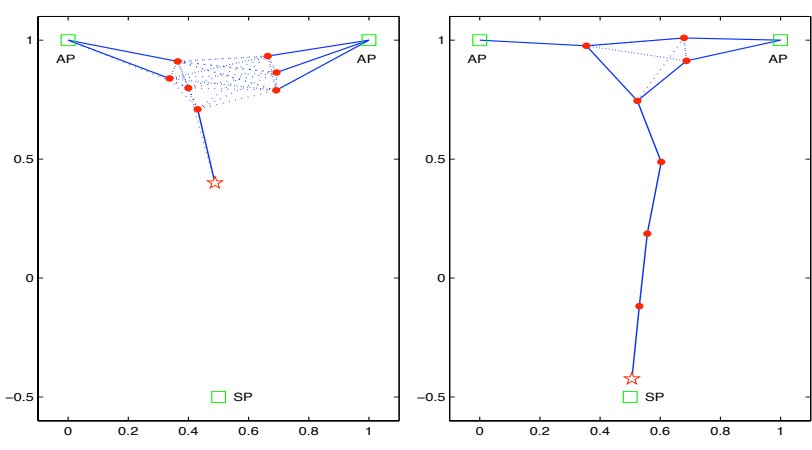

Fig. 2. Communication links are solid or dashed depending on their quality $T_{i j} R_{i j}$, with solid ones indicating higher quality. Packets flow to the APs.

\section{INTEGRATING MOBILITY \& COMMUNICATIONS}

Since mobility introduces nonlinearities in (2), we first propose a centralized hybrid control scheme consisting of continuous-time motion controllers composed with periodic re-optimization of the routing probabilities $T_{i j}$ according to (2). The routing variables are the switching signal in the motion controllers, which rely on artificial potential functions $\phi_{i}=\phi_{i, b}+\phi_{i, c}+\phi_{i, t}$ composed of a barrier potential

$\phi_{i, b}=\left[\left(\sum_{j=1}^{J+K} T_{i j} R_{i j}\right)^{2}-\left(\sum_{j=1}^{J} T_{j i} R_{j i}+r_{i 0}\right)^{2}\right]^{-1}$

that ensures internal consistency of the routing variables $T_{i j}$ at terminal $i$ according to (2), a collision avoidance potential $\phi_{i, c}=\sum_{j \neq i}\left\|\mathbf{x}_{i}-\mathbf{x}_{j}\right\|^{-2}$, and task potentials $\phi_{i, t}$ that can be designed to model a variety of tasks, as it will be discussed bellow. Under mild conditions on the functions $R\left(\mathbf{x}_{i}, \mathbf{x}_{j}\right)$ and $\phi_{i, t}$, we can define a closed loop hybrid system by the integration of the optimization (2) with the motion controllers

$$
\dot{\mathbf{x}}_{i}=-\nabla_{\mathbf{x}_{i}} \phi_{i}, \quad \text { for all } i=1, \ldots, J .
$$

Proposition 1 ( [7]) The closed loop system (2) - (4) guarantees that all robots can communicate with the infrastructure at a basal rate of $r_{i 0}$ packets per unit time. Moreover, collisions between robots are avoided.

To illustrate the proposed framework we consider simple models of channel reliabilities that are deterministic, decreasing functions of the inter-robot distances $\left\|\mathbf{x}_{i j}\right\|=\left\|\mathbf{x}_{i}-\mathbf{x}_{j}\right\|$. One possible choice is $R_{i j}=a\left\|\mathbf{x}_{i j}\right\|^{3}+b\left\|\mathbf{x}_{i j}\right\|^{2}+c\left\|\mathbf{x}_{i j}\right\|+d$ if $l \leq \mathbf{x}_{i j} \leq u, R_{i j}=1$ if $\left\|\mathbf{x}_{i j}\right\|<l$ and $R_{i j}=0$ if $\left\|\mathbf{x}_{i j}\right\|>u$, where $0<l<u$ lower and upper bounds on the inter-robot distances, respectively, and the constants $a$, $b, c$ and $d$ chosen such that $R_{i j}=R\left(\mathbf{x}_{i}, \mathbf{x}_{j}\right)$ is a twice differentiable function ranging from 0 to 1 [7]. We further assume one robot is the leader that is responsible for serving the SP and the other robots relay the information back to the APs. We choose $\phi_{i, t}=\frac{1}{2}\left\|\mathbf{x}_{i}-\mathbf{x}_{i, t}\right\|^{2}$ and $r_{i 0}=.8$ for the leader, and $\phi_{i, t}=0$ and $r_{i 0}=0$ for all relay robots, where $\mathbf{x}_{i, t}$ denotes the location of the service point. In this scenario, the utilities $U_{i}\left(r_{i}\right)=0$ for all robots. Shown in Fig. 2 is the evolution of the system under the influence of the leader. 


\section{Distributed Optimal Communications}

Solving (2) at a central designated node as in Section III entails a large communication cost to convey the network's topology and disseminate the optimal operating point. This cost can be avoided by devising a distributed solution based on the separability of the Lagrangian dual of (2). To do so, associate Lagrange multipliers $\lambda_{i}$ with each of the routing constraints in (1), and define the Lagrangian as

$$
\begin{aligned}
& \mathcal{L}_{\mathbf{x}}(\boldsymbol{\lambda}, \mathbf{T}, \mathbf{r})=\sum_{i=1}^{J} U_{i}\left(r_{i}\right)+\sum_{i=1}^{J} \sum_{j=1}^{J+K} V_{i j}\left(T_{i j}\right) \\
& +\sum_{i=1}^{J} \lambda_{i}\left[\sum_{j=1}^{J+K} T_{i j} R\left(\mathbf{x}_{i}, \mathbf{x}_{j}\right)-\sum_{j=1}^{J} T_{j i} R\left(\mathbf{x}_{j}, \mathbf{x}_{i}\right)-r_{i}\right] .
\end{aligned}
$$

where $\lambda, \mathbf{r} \in \mathbb{R}^{J}$ are vectors of Lagrange multipliers and rates and $\mathbf{T} \in \mathbb{R}^{J \times J+K}$ is a matrix of routing probabilities $T_{i j}$. The dual function is then defined as the maximum of the Lagrangian with respect to primal variables, i.e.,

$$
g_{\mathbf{x}}(\boldsymbol{\lambda})=\max _{r_{i} \geq r_{i 0}, \sum_{j=1}^{J+K} T_{i j} \leq 1} \mathcal{L}_{\mathbf{x}}(\boldsymbol{\lambda}, \mathbf{T}, \mathbf{r}) .
$$

The dual problem is finally defined as the minimization of the dual function, $D_{\mathbf{x}}=\min _{\boldsymbol{\lambda} \geq \mathbf{0}} g_{\mathbf{x}}(\boldsymbol{\lambda})$. Since for fixed spatial configurations $\mathbf{x}$, the problem in (2) is convex it holds that $D_{\mathbf{x}}=P_{\mathbf{x}}$ implying that we can work with the dual problem in lieu of the primal problem in (2). In particular, a distributed algorithm can be obtained by implementing gradient descent in the dual domain.

To implement dual gradient descent we compute the gradient of the dual function using primal Lagrangian maximizers, see e.g., [8]. For given $\boldsymbol{\lambda}$ define the primal Lagrangian maximizers as

$$
\left\{r_{\mathbf{x}, i}(\boldsymbol{\lambda})\right\}_{\forall i},\left\{T_{\mathbf{x}, i j}(\boldsymbol{\lambda})\right\}_{\forall i, j} \triangleq \underset{\substack{r_{i} \geq r_{i 0}, \sum_{j=1}^{J+K} T_{i j} \leq 1}}{\operatorname{argmax}} \quad \mathcal{L}_{\mathbf{x}}(\boldsymbol{\lambda}, \mathbf{T}, \mathbf{r}) .
$$

The components of the dual function's gradient are then given by the constraint slack associated with $\left\{r_{\mathbf{x}, i}(\boldsymbol{\lambda})\right\}_{\forall i}$ and $\left\{T_{\mathbf{x}, i j}(\boldsymbol{\lambda})\right\}_{\forall i, j}$, i.e.,

$$
\begin{aligned}
{\left[\nabla g_{\mathbf{x}}(\boldsymbol{\lambda})\right]_{i}=\sum_{j=1}^{J+K} T_{\mathbf{x}, i j}(\boldsymbol{\lambda}) R\left(\mathbf{x}_{i}, \mathbf{x}_{j}\right) } & \\
& -\sum_{j=1}^{J} T_{\mathbf{x}, j i}(\boldsymbol{\lambda}) R\left(\mathbf{x}_{j}, \mathbf{x}_{i}\right)-r_{\mathbf{x}, i}(\boldsymbol{\lambda}) .
\end{aligned}
$$

A key observation here is that the Lagrangian in (5) can be written as a sum of local Lagrangians that depend only on variables $r_{i}$ and $\left\{T_{i j}\right\}_{\forall i}$. Indeed, it suffices to reorder terms in (5) to realize that upon defining local Lagrangians

$$
\begin{aligned}
\mathcal{L}_{\mathbf{x}, i}(\boldsymbol{\lambda}, \mathbf{T}, \mathbf{r}) & =U_{i}\left(r_{i}\right)-\lambda_{i} r_{i} \\
+ & \sum_{j=1}^{J}\left[V_{i j}\left(T_{i j}\right)+T_{i j} R\left(\mathbf{x}_{i}, \mathbf{x}_{j}\right)\left(\lambda_{i}-\lambda_{j}\right)\right] \\
+ & \sum_{j=J+1}^{J+K}\left[V_{i j}\left(T_{i j}\right)+\lambda_{i} T_{i j} R\left(\mathbf{x}_{i}, \mathbf{x}_{j}\right)\right]
\end{aligned}
$$

it is possible to write

$$
\mathcal{L}_{\mathbf{x}}(\boldsymbol{\lambda}, \mathbf{T}, \mathbf{r})=\sum_{i=1}^{J} \mathcal{L}_{\mathbf{x}, i}(\boldsymbol{\lambda}, \mathbf{T}, \mathbf{r}) .
$$

The local Lagrangian $\mathcal{L}_{\mathbf{x}, i}(\boldsymbol{\lambda}, \mathbf{T}, \mathbf{r})$ is defined so that all summands of the global Lagrangian $\mathcal{L}_{\mathbf{x}}(\boldsymbol{\lambda}, \mathbf{T}, \mathbf{r})$ that involve primal variables $r_{i}$ and $\left\{T_{i j}\right\}_{j=1}^{J+K}$ for given $i$ appear in, and only in, $\mathcal{L}_{\mathbf{x}, i}(\boldsymbol{\lambda}, \mathbf{T}, \mathbf{r})$ [cf. (5) and (9)]. Therefore, to find the variables $r_{\mathbf{x}, i}(\boldsymbol{\lambda})$ and $\left\{T_{\mathbf{x}, i j}(\boldsymbol{\lambda})\right\}_{j=1}^{J+K}$ that maximize the global Lagrangian as per (7) it suffices to find the arguments that maximize the local Lagrangians in (9),

$$
r_{\mathbf{x}, i}(\boldsymbol{\lambda}),\left\{T_{\mathbf{x}, i j}(\boldsymbol{\lambda})\right\}_{j=1}^{J+K}=\underset{\substack{r_{i} \geq r_{i 0}, \sum_{j=1}^{J+K} T_{i j} \leq 1}}{\operatorname{argmax}} \mathcal{L}_{\mathbf{x}, i}(\boldsymbol{\lambda}, \mathbf{T}, \mathbf{r}) .
$$

where the constraints $r_{i} \geq r_{i 0}$ and $\sum_{j=1}^{J+K} T_{i j} \leq 1$ in (11) are for the node $i$ under consideration. Contrast (7) and (11) to observe that in (7) we maximize the global Lagrangian subject to global constraints, while in (11) we maximize local Lagrangians with respect to local constraints.

Introduce now an index $n$ and consider time instants $\left\{t_{n}\right\}_{n=0}^{\infty}$ at which variables are updated. We can use the observation in (11) to write the following distributed gradient descent algorithm for the dual function:

Primal iteration For given Lagrange multipliers $\boldsymbol{\lambda}\left(t_{n}\right)$ and spatial configuration $\mathbf{x}\left(t_{n}\right)$ compute Lagrangian maximizers $r_{i}\left(t_{n}\right)=r_{\mathbf{x}\left(t_{n}\right), i}\left[\boldsymbol{\lambda}\left(t_{n}\right)\right]$ and $T_{i j}\left(t_{n}\right)=T_{\mathbf{x}\left(t_{n}\right), i j}\left[\boldsymbol{\lambda}\left(t_{n}\right)\right]$, defined as in (7), according to (11) as

$$
r_{i}\left(t_{n}\right),\left\{T_{i j}\left(t_{n}\right)\right\}_{j=1}^{J+K}=\underset{\substack{r_{i} \geq r_{i 0}, \sum_{j=1}^{J+K} T_{i j} \leq 1}}{\operatorname{argmax}} \mathcal{L}_{\mathbf{x}\left(t_{n}\right), i}\left(\boldsymbol{\lambda}\left(t_{n}\right), \mathbf{T}, \mathbf{r}\right) .
$$

Dual Iteration. Use the primal variables $r_{i}\left(t_{n}\right)$ and $T_{i j}\left(t_{n}\right)$ in (12) to update the dual variables as

$$
\begin{gathered}
\lambda_{i}\left(t_{n+1}\right)=\left[\lambda_{i}\left(t_{n}\right)-\epsilon\left(\sum_{j=1}^{J+K} T_{i j}\left(t_{n}\right) R\left(\mathbf{x}_{i}\left(t_{n}\right), \mathbf{x}_{j}\left(t_{n}\right)\right)\right.\right. \\
\left.\left.-\sum_{j=1}^{J} T_{j i}\left(t_{n}\right) R\left(\mathbf{x}_{j}\left(t_{n}\right), \mathbf{x}_{i}\left(t_{n}\right)\right)-r_{i}\left(t_{n}\right)\right)\right]^{+}
\end{gathered}
$$

Letting variables $r_{i}\left(t_{n}\right),\left\{T_{i j}\left(t_{n}\right)\right\}_{j=1}^{J+K}$, and $\lambda_{i}\left(t_{n}\right)$ be associated with terminal $i$, the algorithm described by (12) and (13) can be implemented in a distributed manner. The maximization in (12) requires access to local multipliers $\lambda_{i}\left(t_{n}\right)$ and multipliers $\lambda_{j}\left(t_{n}\right)$ from those terminals for which $R\left(\mathbf{x}_{i}\left(t_{n}\right), \mathbf{x}_{j}\left(t_{n}\right)\right) \neq 0$. Likewise, the dual update (13) requires local primal variables $r_{i}\left(t_{n}\right)$ and $\left\{T_{i j}\left(t_{n}\right)\right\}_{j=1}^{J+K}$ as well as neighboring primal variables $\left\{T_{j i}\left(t_{n}\right)\right\}_{j=1}^{J}$ from terminals that communicate directly with $i$.

\section{Distributed Control of MOBILITY \& COMMUNICATIONS}

Let $\dot{\mathbf{x}}_{i}(t)=\mathbf{u}_{i}\left(\mathbf{x}(t) ; \sigma_{i}\left(t_{n}\right)\right)$ for $t \in\left[t_{n}, t_{n+1}\right)$, denote the dynamics of robot $i$ between times $t_{n}$ and $t_{n+1}$, where the function $\mathbf{u}_{i}\left(\mathbf{x}(t) ; \sigma_{i}\left(t_{n}\right)\right)$ denotes the control signal of robot $i$ and $\sigma_{i}\left(t_{n}\right)$ denotes a switching signal that we define as

$$
\sigma_{i}\left(t_{n}\right)=\left\{T_{i j}\left(t_{n}\right)\right\}_{j=1}^{J+K} \cup\left\{T_{j i}\left(t_{n}\right)\right\}_{j=1}^{J} .
$$

The switching signal $\sigma_{i}\left(t_{n}\right)$ contains the routing variables $\left\{T_{i j}\left(t_{n}\right)\right\}_{j=1}^{J+K}$ that are locally available at robot $i$ and routing 
variables $\left\{T_{j i}\left(t_{n}\right)\right\}_{j=1}^{J}$ are received through communication with neighboring terminals. This information allows us to compute the offered rate at time $t$ which we define as

$$
\begin{aligned}
\beta_{i}\left(\mathbf{x}(t) ; \sigma_{i}\left(t_{n}\right)\right)=\sum_{j=1}^{J+K} & T_{i j}\left(t_{n}\right) R\left(\mathbf{x}_{i}(t), \mathbf{x}_{j}(t)\right) \\
& -\sum_{j=1}^{J} T_{j i}\left(t_{n}\right) R\left(\mathbf{x}_{j}(t), \mathbf{x}_{i}(t)\right) .
\end{aligned}
$$

The offered rate $\beta_{i}\left(\mathbf{x}(t) ; \sigma_{i}\left(t_{n}\right)\right)$ is an upper limit on the rates that can be achieved by the system at time $t$ using available routing variables $T_{i j}\left(t_{n}\right)$. In a static setting we are guaranteed that for large enough time index $t_{n}$, it holds $\beta_{i}\left(\mathbf{x}\left(t_{n}\right) ; \sigma_{i}\left(t_{n}\right)\right) \geq r_{i}\left(t_{n}\right) \geq r_{i 0}$. In a dynamic setting this is not necessarily true because routing variables $T_{i j}\left(t_{n}\right)$ are computed for configuration $\mathbf{x}\left(t_{n}\right)$, not $\mathbf{x}(t)$. In this case we want to monitor the offered rates $\beta_{i}\left(\mathbf{x}(t) ; \sigma_{i}\left(t_{n}\right)\right)$ to ensure that they do exceed the basal requirements $r_{i 0}$. In the language of Definition 1 we want to satisfy network integrity at time $t$ using routing variables $T_{i j}\left(t_{n}\right)$ and rates $r_{i}=\beta_{i}\left(\mathbf{x}(t) ; \sigma_{i}\left(t_{n}\right)\right) \geq r_{i 0}$.

Since the distributed primal-dual iteration (12)-(13) developed in Section IV, only ensures feasibility of the primal variables in the limit of a static system, for a given time $t_{n}$, primal iterates $r_{i}\left(t_{n}\right)$ and $T_{i j}\left(t_{n}\right)$ are close to feasible but not necessarily so; i.e., there exists a small error term $e$ for which $\beta_{i}\left(\mathbf{x}\left(t_{n}\right) ; \sigma_{i}\left(t_{n}\right)\right) \geq r_{i}\left(t_{n}\right)-e \geq r_{i 0}-e$. As the system moves from configuration $\mathbf{x}\left(t_{n}\right)$ to $\mathbf{x}(t)$ for $t>t_{n}$, this error may get larger because routes $T_{i j}\left(t_{n}\right)$ were computed for configuration $\mathbf{x}\left(t_{n}\right)$, not for the current spatial arrangement $\mathbf{x}(t)$. This motivates controllers $\mathbf{u}_{i}\left(\mathbf{x}(t) ; \sigma_{i}\left(t_{n}\right)\right)$ based on potential functions

$$
\hat{\phi}_{i}\left(\mathbf{x}(t) ; \sigma_{i}\left(t_{n}\right)\right)=\frac{\gamma_{i}^{k}\left(\mathbf{x}_{i}(t)\right)}{\tilde{\beta}_{i}^{2}\left(\mathbf{x}(t) ; \sigma_{i}\left(t_{n}\right)\right)},
$$

where $k>0$ is a positive constant, $\gamma_{i}: \mathbb{R}^{2} \rightarrow \mathbb{R}_{+}$serves as a goal potential that robot $i$ strives to minimize, and

$$
\tilde{\beta}_{i}\left(\mathbf{x}(t) ; \sigma_{i}\left(t_{n}\right)\right)=\beta_{i}\left(\mathbf{x}(t) ; \sigma_{i}\left(t_{n}\right)\right)-r_{i 0}+e .
$$

measures satisfaction of the approximate network integrity constraint in $\beta_{i}\left(\mathbf{x}\left(t_{n}\right) ; \sigma_{i}\left(t_{n}\right)\right) \geq r_{i 0}-e$ for the communication rate offered to robot $i$ and serves as an obstacle barrier potential that repels the robots from the obstacle set $\tilde{\beta}_{i}\left(\mathbf{x}(t) ; \sigma_{i}\left(t_{n}\right)\right)<0$.

Since $\hat{\phi}_{i}$ can grow unbounded as the approximate network integrity constraints tend to become violated, i.e., as $\tilde{\beta}_{i} \rightarrow 0$, resulting in unbounded robot speeds, we further introduce a diffeomorphism $\psi(y)=\frac{y}{1+y}$ that squashes the image of $\hat{\phi}_{i}$ from $[0, \infty]$ to $[0,1]$. Moreover, define the function $\chi(y)=y^{1 / k}$ to restrict the effect of the obstacles close to the boundary of the free space $\tilde{\beta}_{i}\left(\mathbf{x}(t) ; \sigma_{i}\left(t_{n}\right)\right)=0$. Composition of $\chi, \psi$ and $\hat{\phi}_{i}$ results in the artificial potential

$$
\phi_{i}=\chi \circ \psi \circ \hat{\phi}_{i}=\frac{\gamma_{i}}{\left(\gamma_{i}^{k}+\tilde{\beta}_{i}^{2}\right)^{1 / k}} .
$$

The control law for every robot $i$ can be defined by the negative gradient of the potential $\phi_{i}$ in (17) resulting in

$$
\dot{\mathbf{x}}_{i}(t)=-\alpha \nabla_{\mathbf{x}_{i}} \phi_{i}\left(\mathbf{x}(t) ; \sigma_{i}\left(t_{n}\right)\right),
$$

for all $i=1, \ldots, J$ and all $t \in\left[t_{n}, t_{n+1}\right)$, with $\alpha>0$ a speed gain. The communication variables in $\sigma_{i}\left(t_{n}\right)$ are regulated by the primal-dual iteration (12)-(13) at times $\left\{t_{n}\right\}_{n=0}^{\infty}$.

\section{A. Algorithm Analysis}

To simplify presentation introduce a vector $\mathbf{t}$ stacking the rows of the transmission probability matrix $\mathbf{T}$ and a matrix $\mathbf{A}_{\mathbf{x}}$ with dimensions $J \times J(J+K)$ so as to write the constraints in (1) as $\mathbf{A}_{\mathbf{x}} \mathbf{t}-\mathbf{r} \geq \mathbf{0}$. Using this definition we can rewrite (2) as

$$
P_{\mathbf{x}}=\max f_{0}(\mathbf{r}, \mathbf{t}), \quad \text { s.t. } \mathbf{A}_{\mathbf{x}} \mathbf{t}-\mathbf{r} \geq \mathbf{0},
$$

where the constraints $r_{i} \geq r_{i 0}$ and $\sum_{j=1}^{J+K} T_{i j} \leq 1$ were left implicit. Similarly, we can use this shorthand notation to rewrite the optimal distributed communication algorithm in (12)-(13) as

$$
\begin{aligned}
& \mathbf{t}\left(t_{n}\right), \mathbf{r}\left(t_{n}\right)=\operatorname{argmax} \mathcal{L}_{\mathbf{x}\left(t_{n}\right), i}\left(\boldsymbol{\lambda}\left(t_{n}\right), \mathbf{t}, \mathbf{r}\right), \\
& \boldsymbol{\lambda}\left(t_{n+1}\right)=\left[\boldsymbol{\lambda}\left(t_{n}\right)-\epsilon\left(\mathbf{A}_{\mathbf{x}\left(t_{n}\right)} \mathbf{t}\left(t_{n}\right)-\mathbf{r}\left(t_{n}\right)\right)\right]^{+} .
\end{aligned}
$$

In a static setting, i.e., when the robot positions $\mathbf{x}(t)$ are fixed, e.g., at $\mathbf{x}\left(t_{n}\right)$, for all time $t \geq t_{0}$, it is known that the dual variables $\boldsymbol{\lambda}\left(t_{n}\right)$ approach the optimal multipliers $\boldsymbol{\lambda}_{\mathbf{x}\left(t_{n}\right)}^{*}$. As we already observed, convergence of the primal variables $\mathbf{t}\left(t_{n}\right)$ and $\mathbf{r}\left(t_{n}\right)$ to the optimal network operating point $\mathbf{t}_{\mathbf{x}\left(t_{n}\right)}^{*}, \mathbf{r}_{\mathbf{x}\left(t_{n}\right)}^{*}$ follows provided some technical conditions hold. However when terminals move as per (18), the optimal operating point drifts away towards $\mathbf{t}_{\mathbf{x}\left(t_{n+1}\right)}^{*}, \mathbf{r}_{\mathbf{x}\left(t_{n+1}\right)}^{*}$. Our goal in this section is to determine the optimality of the operating point $\mathbf{t}\left(t_{n}\right), \mathbf{r}\left(t_{n}\right)$ with respect to the optimal operating point $\mathbf{t}_{\mathbf{x}\left(t_{n}\right)}^{*}, \mathbf{r}_{\mathbf{x}\left(t_{n}\right)}^{*}$ for the current team configuration $\mathbf{x}\left(t_{n}\right)$.

Throughout the subsequent analysis we make the following assumptions on the dual functions $g_{\mathbf{x}}(\boldsymbol{\lambda})$ : (A1) The dual functions $g_{\mathbf{x}}(\boldsymbol{\lambda})$ are strongly convex with common strong convexity parameter $m$, i.e., $g_{\mathbf{x}}(\boldsymbol{\mu}) \geq g_{\mathbf{x}}(\boldsymbol{\lambda})+\nabla g_{\mathbf{x}}(\boldsymbol{\lambda})^{T}(\boldsymbol{\mu}-$ $\boldsymbol{\lambda})+\frac{m}{2}\|\boldsymbol{\lambda}-\boldsymbol{\mu}\|^{2}$; (A2) The gradients of the dual functions $g_{\mathbf{x}}(\boldsymbol{\lambda})$ are Lipschitz continuous with common Lipschitz constant $M$, i.e., $\left\|\nabla g_{\mathbf{x}}(\boldsymbol{\lambda})-\nabla g_{\mathbf{x}}(\boldsymbol{\mu})\right\| \leq M\|\boldsymbol{\lambda}-\boldsymbol{\mu}\|$; (A3) The 2-norm of the dual gradients $\nabla g_{\mathbf{x}}(\boldsymbol{\lambda})$ are uniformly bounded for all $\boldsymbol{\lambda}$ and all $\mathbf{x}$, i.e., $\left\|\nabla g_{\mathbf{x}}(\boldsymbol{\lambda})\right\| \leq G_{\max }$; (A4) The 1-norm of the optimal Lagrange multipliers $\lambda_{\mathrm{x}}^{*}$ are uniformly bounded for all $\mathbf{x}$, i.e., $\left\|\boldsymbol{\lambda}_{\mathbf{x}}^{*}\right\|_{1} \leq \lambda_{\max }$. These assumptions are mild, technical, and commonly required in the analysis of gradient descent algorithms. The following result describes the distance between the current $\boldsymbol{\lambda}\left(t_{n}\right)$ and the current optimal $\lambda_{\mathbf{x}\left(t_{n}\right)}^{*}$ Lagrange multipliers.

Theorem 1 ( $[9,10])$ Let $\mathbf{x}\left(t_{n}\right)$ denote the team configuration at iteration $n, \lambda_{\mathbf{x}\left(t_{n}\right)}^{*}$ the corresponding optimal dual variable and $\boldsymbol{\lambda}\left(t_{n}\right)$ the dual iterate obtained through iterative application of (20). Assume the step size in (20) is bounded as $\epsilon \leq 1 / M$ and that the difference between reliabilities at subsequent configurations is absolutely bounded

$$
\left|R\left(\mathbf{x}_{i}\left(t_{n+1}\right), \mathbf{x}_{j}\left(t_{n+1}\right)\right)-R\left(\mathbf{x}_{i}\left(t_{n}\right), \mathbf{x}_{j}\left(t_{n}\right)\right)\right| \leq \delta
$$


for $\delta>0$. If assumptions (A1), (A2), and (A4) hold, the distance between the dual iterate $\boldsymbol{\lambda}\left(t_{n}\right)$ and the optimal multiplier $\boldsymbol{\lambda}_{\mathbf{x}\left(t_{n}\right)}^{*}$ satisfies

$$
\left\|\boldsymbol{\lambda}\left(t_{n}\right)-\boldsymbol{\lambda}_{\mathbf{x}\left(t_{n}\right)}^{*}\right\| \leq \beta^{n}\left\|\boldsymbol{\lambda}\left(t_{0}\right)-\boldsymbol{\lambda}_{\mathbf{x}\left(t_{0}\right)}^{*}\right\|+\sqrt{\frac{2 \lambda_{\max } J}{m(1-\beta)^{2}} \delta},
$$

where the constant $\beta$ is defined as $\beta \triangleq \sqrt{1 /(1+m \epsilon)}$.

Translating the result in Theorem 1 into results regarding primal variables, we obtain the following Corollary.

Corollary $1([9,10])$ With the same hypotheses and definitions of Theorem 1, the norm of the constraint violation can be bounded as

$$
\begin{aligned}
\left\|\left(\mathbf{r}\left(t_{n}\right)-\mathbf{A}_{\mathbf{x}\left(t_{n}\right)} \mathbf{t}\left(t_{n}\right)\right)^{+}\right\| \leq & M \beta^{n}\left\|\boldsymbol{\lambda}\left(t_{0}\right)-\boldsymbol{\lambda}_{\mathbf{x}\left(t_{0}\right)}^{*}\right\| \\
& +\sqrt{\frac{2 M^{2} \lambda_{\max } J}{m(1-\beta)^{2}}} \delta
\end{aligned}
$$

Corollary 1 implies that the constraint violation is essentially uniformly bounded by a term that only depends on problem specific parameters, i.e., the second term on the right-hand-side of (23). Assume now that the channel reliabilities $R\left(\mathbf{x}_{i}(t), \mathbf{x}_{j}(t)\right)$ are purely functions of the inter-robot distances $\left\|\mathbf{x}_{i j}\right\|=\left\|\mathbf{x}_{i}(t)-\mathbf{x}_{j}(t)\right\|$ so that $\max _{i, j}\left\{\left|\frac{d R\left(\mathbf{x}_{i}, \mathbf{x}_{j}\right)}{d\left\|\mathbf{x}_{i j}\right\|}\right|\right\} \leq M_{R}<\infty$. Let also $\max _{i}\left\{\left\|\nabla_{\mathbf{x}_{i}} \phi_{i}\right\|\right\} \leq M_{\phi}<\infty$ denote an upper bound on the maximum robot speed, which can be shown to exist for the proposed artificial potential $\phi_{i}$ in (17) [9]. The following result extends the feasibility guarantees of Theorem 1 and Corollary 1 to all times $t \geq t_{0}$.

Theorem $2\left(\left[\mathbf{9 , 1 0 ] )}\right.\right.$ Define $\Delta t=\max _{n}\left\{t_{n+1}-t_{n}\right\}$ and let $M_{R}$ be the bound on the norm of the gradient of $R\left(\mathbf{x}_{i}, \mathbf{x}_{j}\right)$ and $M_{\phi}$ the bound on robots' velocities. Assume the hypotheses in Theorem 1 hold and that for time $t_{0}$ the communication variables are initialized at the optimal configuration, i.e., $\boldsymbol{\lambda}\left(t_{0}\right)=\boldsymbol{\lambda}^{*}, \mathbf{r}\left(t_{0}\right)=\mathbf{r}_{\mathbf{x}\left(t_{0}\right)}^{*}$, and $\mathbf{T}\left(t_{0}\right)=\mathbf{T}_{\mathbf{x}\left(t_{0}\right)}^{*}$. Then, for any tolerance e satisfying

$$
e>\sqrt{2 \alpha \Delta t M_{R} M_{\phi} \frac{2 M \lambda_{\max } J}{m(1-\beta)^{2}}},
$$

we have $\beta_{i}\left(\mathbf{x}(t) ; \sigma_{i}(t)\right)>r_{i 0}-e$ for all times $t \geq t_{0}$.

Performance of the proposed distributed algorithm, for channel reliabilities similar the ones described in Section III, is shown in Fig. 3. Here the goal potential is $\gamma_{i}=\left\|\mathbf{x}_{i}-\mathbf{x}_{i, t}\right\|^{2}$ for the leader and $\gamma_{i}=1$ for the relays, with $\mathbf{x}_{i, t}$ the location of the service point.

\section{CONCLUSIONS}

In this paper we considered the problem of ensuring communication integrity in networks of mobile robots. Our approach relied on introducing weights on the communication links to capture channel reliabilities, which then allowed to model routing by means of optimization problems that accept

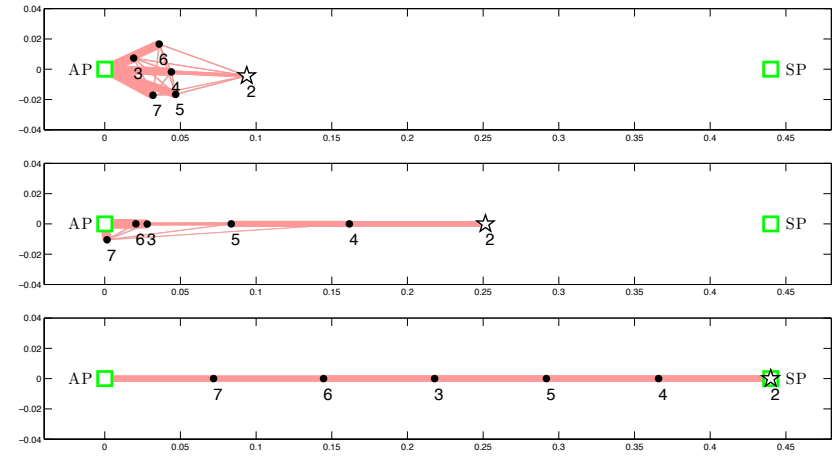

Fig. 3. A mobile robot network consisting of 6 robots (dots) that need to establish reliable communications between one service point (SP) and one access point (AP). The red lines represent routing of information $T_{i j} R\left(\mathbf{x}_{i}, \mathbf{x}_{j}\right)$, between pairs of robots. The thickness of each line represents the magnitude of these quantities. The network involves one leader (star), labeled by the number 2, that moves towards the service point SP.

link reliabilities as inputs. The key idea proposed in this work was the joint control of mobility and communications in a hybrid scheme with the discrete-time routing variables being the switching signal in the continuous-time motion controllers. We developed both centralized and distributed integration schemes, and for the latter case we provided communication guarantees within a bounded error of optimality.

\section{REFERENCES}

[1] K. Pahlavan and A. H. Levesque, Wireless Information Networks. New York, NY: John Willey \& Sons, 1995.

[2] M. Ji and M. Egerstedt, "Coordination control of multi-agent systems while preserving connectedness," IEEE Transactions on Robotics, vol. 23, no. 4, pp. 693-703, August 2007.

[3] M. M. Zavlanos and G. J. Pappas, "Distributed connectivity control of mobile networks," IEEE Transactions on Robotics, vol. 24, no. 6, pp. 1416-1428, December 2008.

[4] H. Lundgren, E. Nordstrom, and C. Tschudin, "The gray zone problem in ieee $802.11 \mathrm{~b}$ based ad hoc networks," ACM SIGMOBILE Mobile Computing and Communications Review, vol. 6, no. 3, pp. 104-105, July 2002.

[5] D. DeCouto, D. Aguayo, J. Bicket, and R. Morris, "A high-throughput path metric for multihop wireless routing," in Proc. ofInternational ACM Conference on Mobile Computing and Networking, San Diego, CA, September 2006, pp. 134-146.

[6] A. Ribeiro, N. D. Sidiropoulos, and G. B. Giannakis, "Optimal distributed stochastic routing algorithms for wireless multihop networks," IEEE Transactions on Wireless Communications, vol. 7, no. 11, pp. 4261-4272, November 2008.

[7] M. M. Zavlanos, A. Ribeiro, and G. J. Pappas, "Mobility and routing control in networks of robots," in Proc. of the 49th IEEE Conference on Decision and Control, Atlanta, GA, December 2010, pp. 75457550.

[8] N. Z. Shor, Minimization Methods for Non-Differentiable Functions. Berlin, Heilderberg, Germany: Springer-Verlag, 1985.

[9] M. M. Zavlanos, A. Ribeiro, and G. J. Pappas, "Network integrity in mobile robotic networks," IEEE Transactions on Automatic Control, September 2011, (submitted).

[10] - "Distributed control of mobility and routing in networks of robots," in Proc. 12th IEEE International Workshop on Signal Processing Advances in Wireless Communications, San Francisco, CA, June 2011. 